Bull. Austral. Math. Soc.

$60 \mathrm{~J} 65,60 \mathrm{~s} 70,93 \mathrm{E} 20$

Vol. 43 (1991) [519-528]

\title{
ON A REFLECTED ORNSTEIN-UHLENBECK PROCESS WITH AN APPLICATION
}

\author{
F.A. Attia
}

The resolvent operator and the moment generating function of a reflected OrnsteinUhlenbeck process are obtained. These results are then applied to determine the long-run average cost and the total expected discounted cost of operating a finite storage system with content-dependent release rate.

\section{INTRODUCTION}

Reflected diffusion processes have been used in physical, biological and engineering models. Specifically such models arise in population dynamics (Ricciardi [9]), in diffusion approximation to queueing systems (Giorno, Nobile and Ricciardi [5]) and in storage models. In storage models it is sometimes desirable to have the content process take only non-negative values. This could be accomplished by setting a reflecting boundary at the zero level (Attia and Brockwell [4]).

The purpose of this study is to investigate a reflected Ornstein-Uhlenbeck (OU) process $\{Z(t), t \geqslant 0\}$ which arises as a solution of a storage equation and to illustrate how these results could be applied in a storage model.

In Section 2 we shall obtain the resolvent operator of $Z$, the moment generating function (mgf) of the first hitting time $T_{y}=\inf \{t \geqslant 0: Z(t)=y\}$ and the expected total occupation time of $[0, y], y \in(0, \infty)$. These results are then used via a limiting procedure to obtain explicit expressions for the cost and reward functionals that arise in Section 3. In that section we shall obtain the long-run average cost and the expected total discounted cost of operating a finite dam with content dependent release rate. These results modify and extend those obtained by Zuckerman [10], Lam Yeh [8] and Attia [3].

\section{Received 28 June 1990}

I would like to thank the Department of Mathematical Sciences, Portland State University, Oregon, U.S.A. for its hospitality during my visit. Research carried out while on sabbatical leave from Kuwait University. Research partially supported by Kuwait University Research Grant No. SM 050 and KFAS Grant No. 87-09-01.

Copyright Clearance Centre, Inc. Serial-fee code: 0004-9729/91 \$A2.00+0.00. 


\section{A Reflected Ornstein-Uhlenbeck Process}

The stochastic differential equation

$$
d Z(t)=d X(t)-r(Z(t))
$$

is encountered in storage modelling where $X(t)$ is the input in $(0, t], Z(t)$ is the content and $r(Z(t))$ is the content-dependent release rate at time $t$.

In this section we shall consider $X(t), t \geqslant 0$ to be a Wiener process with drift $\mu$ and variance parameter $\sigma^{2}$ reflected at 0 . Also, we shall assume that $r(\cdot)$ is a linearly increasing function. Thus the above storage equation is of the form

$$
d Z(t)=d X(t)-k Z(t),
$$

where $k$ is a positive constant. If $Z(0)=z$, then an argument generalising the one used by Karlin and Taylor [7, p.345] shows that the solution of the differential equation (2.1), that is,

$$
Z(t)=z e^{-z t}+X(t)-k \int_{0}^{t} e^{-k(t-u)} X(u) d u
$$

is the Ornstein-Uhlenbeck (OU) process with drift $-k z+\mu$ and variance parameter $\sigma^{2}$ reflected at 0 .

The transition semi-group of the process $Z(t)$ is characterised by its generator

$$
\mathcal{A} f(z)=\frac{1}{2} \sigma^{2} f^{\prime \prime}(z)-(k z-\mu) f^{\prime}(z)
$$

where the domain $\mathcal{A}$ is

$$
\mathcal{D}(\mathcal{A})=\left\{f \in C^{2}[0, \infty): f^{\prime}(0)=0\right\}
$$

and $C^{2}$ is the class of twice continuously differentiable functions on $[0, \infty)$.

THE RESOLVENT OPERATOR OF $Z$.

The resolvent operator of a process $Z$ is defined by

$$
\begin{aligned}
\varphi_{\alpha}(z): & =R_{\alpha} f(z) \\
& =E_{z} \int_{0}^{\infty} e^{-\alpha t} f(Z(t)) d t
\end{aligned}
$$

where $E_{z}$ is the expectation conditional on $Z(0)=z$. To determine $R_{\alpha} f(z)$, we note that $\varphi_{\alpha}(z) \in \mathcal{D}(\mathcal{A})$ and thus satisfies the resolvent equation

$$
\begin{gathered}
\mathcal{A} \varphi_{\alpha}(z)-\alpha \varphi_{\alpha}(z)=-f(z) \\
\varphi_{\alpha}^{\prime}(0)=0 \text { and } \varphi_{\alpha}(z) \text { is bounded on }[0, \infty) .
\end{gathered}
$$

where 
Now, expressions (2.3) and (2.4) give the differential equation

$$
\varphi_{\alpha}^{\prime \prime}(z)-\left(2(k z-\mu) / \sigma^{2}\right) \varphi_{\alpha}^{\prime}(z)-\left(2 \alpha / \sigma^{2}\right) \varphi_{\alpha}(z)=-\left(2 / \sigma^{2}\right) f(z)
$$

The homogeneous differential equation in (2.5) has the two independent solutions (Kamke [6, p.416])

and

$$
\varphi_{\alpha, 1}(\xi)=1+\sum_{n=1}^{\infty} \frac{\alpha(\alpha+2 k) \ldots(\alpha+2 k(n-1))}{k^{n}(2 n) !} \xi^{2 n}
$$

$$
\varphi_{\alpha, 2}(\xi)=\xi+\sum_{n=1}^{\infty} \frac{(\alpha+k)(\alpha+3 k) \ldots(\alpha+k(2 n-1))}{k^{n}(2 n+1) !} \xi^{2 n+1}
$$

where

$$
\xi \equiv \xi(z)=\sqrt{2 k}(z-\mu / k) / \sigma .
$$

We note that $\varphi_{\alpha, 1}(y)$ and $\varphi_{\alpha, 2}(y)$ have the following Taylor expansions in the neighbourhood of $\alpha=0$ :

$$
\varphi_{\alpha, 1}(\xi)=\sum_{n=1}^{\infty} a_{n}(\xi) \alpha^{n} \quad \text { and } \quad \varphi_{\alpha, 2}(\xi)=\sum_{n=1}^{\infty} b_{n}(\xi) \alpha^{n},
$$

where for example

$$
a_{0}(\xi)=1 \text { and } b_{0}(\xi)=\xi+\frac{1}{3 !} \xi^{3}+\frac{1.3}{5 !} \xi^{5}+\frac{1.3 .5}{7 !} \xi^{7}+\ldots
$$

The coefficient $b_{0}(\xi)$ is related to Kummer's function (Abramowitz and Stegun [1, p.504]) by

where

$$
\begin{gathered}
b_{0}(\xi)=\xi M\left(\frac{1}{2}, \frac{3}{2}, \frac{\xi^{2}}{2}\right), \\
M(u, v, z)=\sum_{n=1}^{\infty} \frac{(u)_{n}}{(v)_{n}} \frac{z^{n}}{n !}, \\
(u)_{n}=u(u+1) \ldots(u+n-1) \text { and } u_{0}=1 .
\end{gathered}
$$

Finally, a solution of (2.5) which could be extended such that $\varphi_{\alpha}(x) \in \mathcal{D}(\mathcal{A})$ is

$$
\begin{aligned}
R_{\alpha} f(z)= & \varphi_{\alpha, 1}(\xi(z))\left[1+\delta\left(0, z ; \varphi_{\alpha, 2}(\xi(.)), f(.)\right)\right] \\
& -\varphi_{\alpha, 2}(\xi(z)) \delta\left(0, z ; \varphi_{\alpha, 1}(\xi(.)), f(.)\right)
\end{aligned}
$$


where $\xi($.$) is given by (2.6) and$

$$
\delta(x, y ; h(\xi(.)), f(.))=\int_{x}^{y} \exp \left\{-\xi^{2}(u) / 2\right\} h(\xi(u)) f(u) d u .
$$

THE MGF OF $T_{y}$.

Let $g($.$) be a measurable function on [0, \infty)$. Now, Dynkin's formula (see for example $[7, \mathrm{p} .297])$ applied to the Markov time $T_{y}$ gives

$$
E_{x} \int_{0}^{T_{y}} e^{-\alpha t} g(Z(t)) d t=R_{\alpha} g(x)-R_{\alpha} g(y) E_{x}\left[\exp \left\{-\alpha T_{y}\right\}\right], x \leqslant y,
$$

where $E_{x}$ is the expectation conditional on $Z(0)=x$. From expression (2.10) it follows immediately that the moment generating function of $T_{y}$ is given by

$$
E_{x}\left[\exp \left\{-\alpha T_{y}\right\}\right]=\frac{1-\alpha R_{\alpha} I_{y}(x)}{1-\alpha R_{\alpha} I_{y}(y)},
$$

where $I_{y}():.=I_{[0, y]}($.$) is the indicator function of the set [0, y]$. From (2.8), we note that

$$
\begin{aligned}
R_{\alpha} I_{y}(x)= & \varphi_{\alpha, 1}(\xi(x))\left[1+\delta\left(0, y ; \varphi_{\alpha, 2}(\xi(.)), 1(.)\right)\right] \\
& -\varphi_{\alpha, 2}(\xi(x)) \delta\left(0, y ; \varphi_{\alpha, 1}(\xi(.)), 1(.)\right),
\end{aligned}
$$

where $1($.$) is the identity function.$

To obtain the conditional expectation of $T_{y}$ we proceed as follows. First we combine (2.10) and (2.11), then we appeal to the monotone convergence theorem to obtain

$$
E_{x} \int_{0}^{T_{y}} g(Z(t)) d t=\lim _{\alpha \downarrow 0}\left[R_{\alpha} g(x)-R_{\alpha} g(y)\right]
$$

If we now substitute from (2.8) into (2.13), we have

$$
\begin{aligned}
E_{x} \int_{0}^{T_{y}} g(Z(t)) d t= & \delta\left(0, x ; b_{0}(\xi(.)), g(.)\right)-b_{0}(\xi(x)) \delta(0, x ; 1(.), g(.)) \\
& -\delta\left(0, y ; b_{0}(\xi(.)), g(.)\right)+b_{0}(\xi(y)) \delta(0, y ; 1(.), g(.)) .
\end{aligned}
$$

Finally, $E_{x} T_{y}$ follows immediately from the above expression by replacing the function $g($.$) by 1($.$) .$

The EXPECTED OCCUPATION TIME of $[0, y]$.

For $x, y \in[0, \infty), x \leqslant y$, expressions (2.12) and (2.7) give

where

$$
\begin{gathered}
R_{\alpha} I_{y}(x)=\sum_{n=0}^{\infty} c_{n}(\xi(x)) \alpha^{n}, \\
c_{0}(\xi(x))=1+\delta\left(0, y ; b_{0}(\xi(.)), 1(.)\right)-b_{0}(\xi(x)) \delta(0, y ; 1(.), 1(.))
\end{gathered}
$$

is the expected total occupation time of $[0, y]$. 


\section{APplication to a storage MODEL With CONTENT-DEPENDENT RELEASE RATE}

Consider a finite storage system, for example a dam with capacity a. We shall assume that the dam is initially empty. The input to the dam in the time interval $[0, t], X(t)$ is a Wiener process with drift $\mu$ and variance parameter $\sigma^{2}$ with reflecting boundaries at 0 and $a$. In this section we shall determine the long-run average cost per unit time and the total expected discounted cost of operating the storage system.

We shall consider the following optimal output policy in the class of monotone policies $P_{\lambda, \tau}^{M}$. The release rate is kept at 0 until the content level reaches $\lambda(0<\lambda<a)$ at which instant the water is released at a content-dependent rate $r(Z(t))$ with cost $K r(\lambda)$, where $K$ is a positive constant. As long as the content level is more than $\tau(0<\tau<\lambda)$, the output is kept at the rate $r(Z(t))$. When the content reaches $\tau$, the output rate is then decreased to zero at no extra cost (we note here that adding a constant shut-off cost per unit of water released is a trivial extension to our model). Rewards are collected at an output-dependent rate $w(r(Z(t)))$. We also consider a penalty cost which accrues continuously at a rate $g(Z(t))$. Here the functions $g$ and $w$ are bounded measurable functions with support $[0, a]$. In what follows we shall consider the release rate $r($.$) to be a linearly increasing function of the content. Thus$ $r(z)=k z, z>0$ and $r(0)=0$, where $k$ is a positive constant.

(A) The Long-RUn aVerage cost. If there is no release from the dam, the content process $Z(t)$ is the process $X(t)$ killed at $T_{\lambda}$, that is, $Z(t)$ is a Wiener process with drift $\mu$ and variance parameter $\sigma^{2}$ and reflecting boundary at 0 . The transition probabilities of $Z(t)$ are defined in terms of the generator

$$
\mathcal{G} f(z)=\frac{1}{2} \sigma^{2} f^{\prime \prime}(z)+\mu f^{\prime}(z), z \in[0, a],
$$

where the domain of $\mathcal{G}$ is

$$
\mathcal{D}(\mathcal{G})=\left\{f \in C^{2}[0, a]: f^{\prime}(0)=0\right\} .
$$

When there is a content-dependent release rate $r($.$) from the dam, the content$ process satisfies the storage equation (2.1). In the case when $r(z)=k z$ and $Z(0)=z$, the solution of the stochastic differential equation (2.1) is the OU process (2.2).

Now, let us define the following stopping times for the content process $Z(t)$ :

and

$$
\begin{gathered}
T_{0}=\inf \{t \geqslant 0: Z(t)=\lambda\}, T_{0}^{\prime}=\inf \left\{t>T_{0}: Z(t)=\tau\right\}, \\
T_{n}=\inf \left\{t>T_{n-1}^{\prime}: Z(t)=\lambda\right\}, n=1,2, \ldots
\end{gathered}
$$

$$
T_{n}^{\prime}=\inf \left\{t>T_{n}: Z(t)=\tau\right\}, n=1,2, \ldots
$$


The successive returns of $Z$ to the state $\tau$, that is, the Markov times $T_{0}^{\prime}, T_{1}^{\prime}, \ldots$ form a sequence of delayed regeneration times for $Z$.

Clearly, when there is no release from the dam the cost due to the penalty function $g($.$) is$

$$
C_{0}(\tau, \lambda)=E_{\tau} \int_{0}^{T_{\lambda}} g(Z(t)) d t
$$

On the other hand, when $r(z)=k z$ the cost and the income functionals are given respectively by

$$
\begin{aligned}
& C_{r}(\lambda, \tau)=E_{a-\lambda} \int_{0}^{T_{a-\tau}} g\left(Z^{*}(t)\right) d t \\
& R_{r}(\lambda, \tau)=E_{a-\lambda} \int_{0}^{T_{a-\tau}} w\left(k Z^{*}(t)\right) d t
\end{aligned}
$$

where $Z^{*}(t)$ is the process (2.2) killed at $T_{a-\tau}$, that is, $Z^{*}(t)$ is an OU process with drift $-k z+\mu, z \in[0, a]$ and variance parameter $\sigma^{2}$ reflected at 0 . It is obvious that $C_{r}(\lambda, \tau)-R_{r}(\lambda, \tau)$ is the net cost when the release rate from the dam is non-zero.

Using a renewal argument we can show that the long-run average cost per unit time of operating the dam under the $P_{\lambda, \tau}^{r}$ policy is given by

$$
C(\tau, \lambda)=\frac{k \lambda K-R_{r}(\lambda, \tau)+C_{0}(\tau, \lambda)+C_{r}(\lambda, \tau)}{E_{\lambda} T_{\tau}+E_{\tau} T_{\lambda}}
$$

It is clear that $C(\tau, \lambda)$ as given by (4.3) of [2] is a special case of (3.3) for $r()=$.$M , a$ constant and $w \equiv 1$. In [2] we obtained the following expressions for the cost functional $C_{0}(\tau, \lambda)$ and for the conditional expectation of the hitting time $E_{\tau} T_{\lambda}$ :

$$
C_{0}(\tau, \lambda)=(1 / \mu) \int_{0}^{\lambda} g(s)\left[\exp \left\{-\beta(\tau-s)_{+}\right\}-\exp \left\{-\beta(\lambda-s)_{+}\right\}\right] d s
$$

and

$$
E_{\tau} T_{\lambda}=((\lambda-\tau) / \mu)+(1 / \beta \mu)[\exp \{-\beta \lambda\}-\exp \{-\beta \tau\}]
$$

where $x_{+}$is the positive part of $x$ and $\beta=2 \mu / \sigma^{2}$.

To evaluate $C_{r}(\lambda, \tau)$ as given by (3.1), we apply Dynkin's formula to the Markov time $T_{a-T}$ to obtain

$$
\begin{aligned}
& E_{a-\lambda} \int_{0}^{T_{\alpha-\tau}} e^{-\alpha t} g\left(Z^{*}(t)\right) d t \\
& \quad=R_{\alpha}^{*} g(a-\lambda)-R_{\alpha}^{*} g(a-\tau) E_{a-\lambda} \exp \left\{-\alpha T_{a-\tau}\right\}
\end{aligned}
$$

where

$$
E_{a-\lambda} \exp \left\{-\alpha T_{a-\tau}\right\}=\frac{1-\alpha R_{\alpha}^{*} I_{a-\tau}(a-\lambda)}{1-\alpha R_{\alpha}^{*} I_{a-\tau}(a-\tau)}
$$


The operator $\varphi_{\alpha}^{*}=R_{\alpha}^{*} g$ now satisfies the differential equation (2.5) with the boundary conditions

$$
\varphi_{\alpha}^{*^{\prime}}=0 \text { and } \varphi_{\alpha}^{*}(a-\tau)=0 .
$$

The general solution of the differential equation (2.5) with the above conditions is

$$
\begin{aligned}
R_{\alpha}^{*} g(z)= & \beta_{12}(\xi(z), \xi(a-\tau)) \delta\left(0, z ; \varphi_{\alpha, 1}(\xi(.)), g(.)\right) \\
& +\varphi_{\alpha, 1}(\xi(z)) \delta\left(z, a-\tau ; \beta_{12}(\xi(.), \xi(a-\tau)), g(.)\right)
\end{aligned}
$$

where

$$
\beta_{12}(\xi(x), \xi(y))=\left[\varphi_{\alpha, 1}(\xi(x)) \varphi_{\alpha, 2}(\xi(y))-\varphi_{\alpha, 2}(\xi(x)) \varphi_{\alpha, 1}(\xi(y))\right] / \varphi_{\alpha, 1}(\xi(y))
$$

and $\delta$ is defined by (2.9).

We note from (2.7) and (3.6) that $R_{\alpha}^{*} g(z)$ has the following Taylor expansion in the neighbourhood of $\alpha=0$ :

$$
R_{\alpha}^{*} g(z)=\sum_{n=0}^{\infty} c_{n}^{*}(\xi(z)) \alpha^{n}
$$

where for example

$$
\begin{gathered}
c_{0}^{*}(\xi(z))=\left[b_{0}(\xi(a-r))-b_{0}(\xi(z))\right] \delta(0, z ; 1(.), g(.)) / b_{0}(\xi(a-\tau)) \\
+\delta(z, a-\tau ; 1(.), g(.))-\delta\left(z, a-\tau ; b_{0}(\xi(.)), g(.)\right) / b_{0}(\xi(a-\tau)) .
\end{gathered}
$$

For $x, y \in[0, a]$ and $x \leqslant y$ we have from (3.6)

$$
\begin{gathered}
R_{\alpha}^{*} I_{y}(x)=\beta_{12}(\xi(x), \xi(a-\tau)) \delta\left(0, y ; \varphi_{\alpha, 1}(\xi(.)), 1(.)\right) \\
+\varphi_{\alpha, 1}(\xi(x)) \delta\left(x, y ; \beta_{12}(\xi(.), \xi(a-\tau)), 1(.)\right) .
\end{gathered}
$$

If we now combine (3.4)-(3.7) and (3.1) we obtain the cost functional

$$
C_{\tau}(\lambda, \tau)=\lim _{\alpha \downarrow 0}\left[R_{\alpha}^{*} g(a-\lambda)-R_{\alpha}^{*} g(a-\tau)\right]
$$

Appealing to the monotone convergence theorem and utilising (3.7) and (3.8), we have

$$
\begin{aligned}
C_{\mathrm{r}}(\lambda, \tau)= & {\left[b_{0}(\xi(a-\tau))-b_{0}(\xi(a-\lambda))\right] \delta(0, a-\lambda ; 1(.), g(.)) / b_{0}(\xi(a-\tau)) } \\
& +\delta(a-\lambda, a-\tau ; 1(.), g(.))-\delta\left(a-\lambda, a-\tau ; b_{0}(\xi(.)), g(.)\right) / b_{0}(\xi(a-\tau)) .
\end{aligned}
$$

If we replace the function $g($.$) by 1($.$) and by w(r()$.$) , we obtain explicit expressions for$ $E_{\lambda} T_{\tau}$ and for $R_{r}(\lambda, \tau)$ respectively. This completes the determination of the long-run average cost per unit time of operating the storage system under a $P_{\lambda, \tau}^{M}$ policy. The cost $C(\tau, \lambda)$ can then be minimised with respect to $\tau$ and $\lambda$. 
(B) The expected total Discounted cost. Following Attia [2] and Zuckerman [10], the expected discounted cost incurred when the dam is operated under the $P_{\lambda, r}^{r}$ policy is given by

$$
C_{\alpha}(\tau, \lambda)=E_{0} \sum_{n=0}^{\infty} C_{n, \alpha}(\tau, \lambda), 0<\alpha<1,
$$

where $C_{n, \alpha}(\tau, \lambda)$ is the cost incurred during the time interval $\left[T_{n-1}, T_{n}\right), n=1,2, \ldots$ and $C_{0}(\tau, \lambda)$ is the cost incurred in the interval $\left[0, T_{0}\right)$. A simple renewal argument together with the strong Markov property applied to expression (3.11) gives

Clearly

$$
\begin{gathered}
C_{\alpha}(\tau, \lambda)=E_{0} C_{0, \alpha}(\tau, \lambda)+\frac{E_{0} \exp \left(-\alpha T_{\lambda}\right) E_{\lambda} C_{1, \alpha}(\tau, \lambda)}{1-E_{\lambda} \exp \left(-\alpha T_{\tau}\right) E_{\tau} \exp \left(-\alpha T_{\lambda}\right)} \\
E_{0} C_{0, \alpha}(\tau, \lambda)=E_{0} \int_{0}^{T_{\lambda}} e^{-\alpha t} g(Z(t)) d t
\end{gathered}
$$

Now, using the strong Markov property, the expected discounted cost over the first cycle could be written in the form

$$
\begin{gathered}
E_{\lambda} C_{1, \alpha}(\tau, \lambda)=k \lambda K+E_{a-\lambda} \int_{0}^{T_{a-\tau}} e^{-\alpha t} g\left(Z\left(^{*}(t)\right)\right) d t \\
+E_{\lambda} e^{-\alpha T_{\tau}} E_{\tau} \int_{0}^{T_{\lambda}} e^{-\alpha t} g(Z(t)) d t-E_{a-\lambda} \int_{0}^{T_{a-\tau}} e^{-\alpha t} w\left(k Z^{*}(t)\right) d t .
\end{gathered}
$$

In a recent paper [2] we have shown that, for the reflected Wiener process $Z(t)$ and for any bounded measurable function $f$ with support $[0, y]$,

$$
\begin{gathered}
E_{x} \int_{0}^{T_{y}} \exp (-\alpha t) f(Z(t)) d t=R_{\alpha} f(x)-R_{\alpha} f(y) \frac{1-\alpha R_{\alpha} I_{y}(x)}{I-\alpha R_{\alpha} I_{y}(y)} \\
x<y
\end{gathered}
$$

where the resolvent operator of the process $Z(t)$ could be expressed in the form

$$
\begin{aligned}
R_{\alpha} f(x) & =E_{x} \int_{0}^{\infty} e^{-\alpha t} f(Z(t)) d t \\
& =\int_{0}^{a} K_{\alpha}(x, y) f(y) d y
\end{aligned}
$$

the associated kernel $K_{\alpha}(x, y)$ is given by (see Attia [3])

$$
\begin{aligned}
& K_{\alpha}(x, y)=\frac{1}{\gamma} \exp \left\{\mu(y-x) / \sigma^{2}\right\} \\
& \quad \times\left[\frac{\gamma-\mu}{\gamma+\mu} \exp \left\{-\gamma(y+x) / \sigma^{2}\right\}+\exp \left\{-\gamma|y-x| / \sigma^{2}\right\}\right], \quad x, y \in[0, a]
\end{aligned}
$$


and $\gamma=\left(\mu^{2}+2 \alpha \sigma^{2}\right)^{1 / 2}$.

It is straightforward to show that (3.14) and (3.15) give

$$
\begin{aligned}
R_{\alpha} f(x)= & \frac{1}{\gamma} \exp \left\{-x(\mu+\gamma) / \sigma^{2}\right\}\left[\frac{\gamma-\mu}{\gamma+\mu} \int_{0}^{a} \exp \left\{-(\gamma-\mu) y / \sigma^{2}\right\} f(y) d y\right. \\
& \left.+\int_{0}^{x} \exp \left\{(\gamma+\mu) y / \sigma^{2}\right\} f(y) d y\right] \\
& +\frac{1}{\gamma} \exp \left\{-x(\mu-\gamma) / \sigma^{2}\right\} \int_{x}^{a} \exp \left\{-(\gamma-\mu) y / \sigma^{2}\right\} f(y) d y
\end{aligned}
$$

Now, if we substitute $f \equiv 1$ in (3.13), carry out the integration and then use (3.16), we get

$$
\begin{aligned}
E_{x} \exp \left\{-\alpha T_{y}\right\} & =\frac{1-\alpha R_{\alpha} I_{y}(x)}{1-\alpha R_{\alpha} I_{y}(y)} \\
& =\frac{(\gamma+\mu) \exp \left\{(\gamma-\mu) x / \sigma^{2}\right\}+(\gamma-\mu) \exp \left\{-(\gamma+\mu) x / \sigma^{2}\right\}}{(\gamma+\mu) \exp \left\{(\gamma-\mu) y / \sigma^{2}\right\}+(\gamma-\mu) \exp \left\{-(\gamma+\mu) y / \sigma^{2}\right\}}, x<y
\end{aligned}
$$

Expressions for $E_{0}\left[\exp \left\{-\alpha T_{\lambda}\right\}\right]$ and $E_{r}\left[\exp \left\{-\alpha T_{\lambda}\right\}\right]$ immediately follow from (3.13). Substituting from (3.16) and (3.17) with the proper values for $x$ and $y$ gives explicit expressions for $E_{0} C_{0, \alpha}(\lambda, \tau)$ and for $E_{\tau} \int_{0}^{T_{\lambda}} \exp \{-\alpha t\} g(Z(t)) d t$.

Expressions similar to (3.13) and (3.14) hold also for the reflected OU process $Z^{*}(t)$. An expression corresponding to (3.17) for $E_{\lambda}\left[\exp \left\{-\alpha T_{\tau}\right\}\right]$ is obtained by combining (3.5) and (2.12) with the proper modifications. If we now substitute from (2.8) and (2.12) in (3.13) with the obvious modifications, we obtain an explicit expression for

$$
E_{a-\lambda} \int_{0}^{T_{a-\tau}} \exp (-\alpha t) g\left(Z^{*}(t)\right) d t .
$$

An explicit expression for the discounted reward functional

$$
E_{a-\lambda} \int_{0}^{T_{a-\tau}} \exp (-\alpha t) w\left(k Z^{*}(t)\right) d t
$$

could be obtained when $w($.$) is the polynomial function w(z)=\sum_{i=0}^{n} e u_{i} z^{i}$. In this case

$$
\begin{aligned}
E_{a-\lambda} & \int_{0}^{T_{a-\tau}} \exp (-\alpha t) w\left(k Z^{*}(t)\right) d t \\
& =\sum_{i=0}^{n} k e u_{i} E_{a-\lambda} \int_{0}^{T_{a-\tau}} e^{-\alpha t}\left(Z^{*}(t)\right)^{i} d t .
\end{aligned}
$$


Dynkin's lemma applied to the Markov time $T_{a-r}$ give

$$
E_{a-\lambda} \int_{0}^{T_{a-\tau}} e^{-\alpha t}\left(Z^{*}(t)\right)^{n} d t=R_{\alpha}^{*} I^{n}(a-\lambda)-R_{\alpha}^{*} I^{n}(a-\tau) E_{a-\lambda}\left\{\exp \left(-\alpha T_{a-\tau}\right)\right\}
$$

where $I^{n}($.$) is the n$th power function. This completes the determination of the expected total discounted cost as given by (3.12).

Finally, we note that

$$
\begin{aligned}
\lim _{\alpha \rightarrow 0+} \alpha C_{\alpha}(\tau, \lambda) & =\left[1 /\left(E_{\lambda} T_{\tau}+E_{\tau} T_{\lambda}\right)\right]\left[k \lambda K-E_{a-\lambda} \int_{0}^{T_{a-\lambda}} w\left(k Z^{*}(t)\right) d t\right. \\
& \left.+E_{\tau} \int_{0}^{T_{\lambda}} g(Z(t)) d t+E_{a-\lambda} \int_{0}^{T_{a-\tau}} g\left(Z^{*}(t)\right) d t\right]
\end{aligned}
$$

which is precisely the long-run average cost per unit time of operating the storage system when policy $P_{\lambda_{\tau}}^{r}$ is employed.

\section{REFERENCES}

[1] M. Abramowitz and I.A. Stegun, Handbook of mathematical functions with formulas, graphs and mathematical tables (Dover, New York, 1965).

[2] F.A. Attia, 'A note on the expected discounted cost of operating a finite dam', Stochastic Process. Appl. 31 (1989), 161-166.

[3] F.A. Attia, 'The control of a finite dam with penalty cost function: Wiener Process input', Stochastic Process. Appl. 25 (1987), 289-299.

[4] F.A. Attia and P.J. Brockwell, 'The control of a finite dam', J. Appl. Probab. 1 (1982), 815-825.

[5] V. Giorno, A.G. Nobile and L.M. Ricciardi, 'On some diffusion approximations to queueing systems', Adv. in Appl. Probab. 18 (1986), 991-1014.

[6] E. Kamke, Differentialgleichungen Lösungsmethoden and Lösungen (Chelsea, New York, 1971).

[7] S. Karlin and H.M. Taylor, A second course in stochastic processes (Academic Press, New York, 1981).

[8] Lam Yeh, 'Optimal control of a finite dam: Average cost case', J. Appl. Probab. 22 (1985), 480-484.

[9] L.M. Ricciardi, Stochastic population models II. Diffusion models: Lecture Notes at the International School on Mathematical Ecology, 1985.

[10] D. Zuckerman, 'Two-stage output procedure of a finite dam', J. Appl. Probab. 14 (1977), 421-425. 